\title{
A IMPORTÂNCIA DA AUDITORIA NA MANUTENÇÃO DOS PROCESSOS EM DISTRIBUIDORAS DE MATERIAL MÉDICO HOSPITALAR (MMH)
}

\section{THE IMPORTANCE OF AUDITING IN THE MAINTENANCE OF PROCESSES IN DISTRIBUTORS OF HOSPITAL MEDICAL MATERIAL (HMM)}

Paulo Augusto Bandeira Bernardino¹, Aline André da Silva Pereira², Amanda Gonçalves Pereira ${ }^{3}$

\author{
Submetido: 01/12/2020 \\ Aprovado: 16/01/2021
}

\section{RESUMO}

A auditoria atua diretamente na manutenção dos processos exigidos pela RDC 16/2013 publicada pela ANVISA em 2013. A norma oferece requisitos de boas práticas para as distribuidoras de $\mathrm{MMH}$ que, consequentemente, seguidos de forma concreta impacta positivamente no resultado do produto e serviço oferecidos, visando a saúde do paciente e sua qualidade de vida. A auditoria é uma das maneiras eficazes de identificar não conformidades, oportunidades de melhorias e monitoramento das atividades realizadas, atendendo as especificações estabelecidas na norma. Com o controle e acompanhamento periódico realizado através dos programas de auditoria obtém-se um aprimoramento dos desempenhos garantindo um atendimento de qualidade que gera diferencial competitivo.

PALAVRAS-CHAVE: Auditoria. ANVISA. RDC 16/2013. Qualidade. Boas Práticas. Melhorias.

\begin{abstract}
The audit acts directly in the maintenance of the processes required by RDC 16/2013 published by ANVISA in 2013. The standard offers good practice requirements for $\mathrm{MMH}$ distributors that, consequently, followed in a concrete way positively impacts on the result of the product and service offered, aiming at the health of the patient and their quality of life. Auditing is one of the effective ways to identify non-conformities, opportunities for improvement and monitoring of the activities carried out, meeting the specifications set forth in the standard. With the periodic control and monitoring performed through audit programs, performance improvement is achieved, ensuring quality care that generates a competitive differential.
\end{abstract}

KEYWORDS: Audit. ANVISA. RDC 16/2013. Quality. Good Practices. Improvements.

\section{INTRODUÇÃO}

O tema que será tratado nesse artigo refere-se à importância da auditoria interna nos distribuidores de Material Médico Hospitalar ( $\mathrm{MMH}$ ) que realizam a ponte entre fabricantes e hospitais, atendendo às demandas de cirurgias, sejam elas eletivas ou de emergência. No Brasil a legislação pertinente às distribuidoras é a Resolução da Diretoria Colegiada - RDC 16 (ANVISA, 2013). Essa resolução oferece requisitos de boas práticas relativos à produção, comercialização e controle do $\mathrm{MMH}$ e dos

\footnotetext{
${ }_{1}^{1}$ Mestre em Educação e Bacharel em Filosofia pela Universidade Federal de Minas Gerais. (UFMG). Docente na Faculdade Minas Gerais - Famig

${ }^{2}$ Graduanda em Administração de Empresas na Faculdade Minas Gerais - Famig

3 Graduanda em Administração de Empresas na Faculdade Minas Gerais - Famig
} 


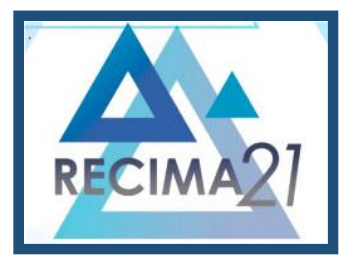

\section{RECIMA21 - REVISTA CIENTÍFICA MULTIDISCIPLINAR}

A IMPORTÂNCIA DA AUDITORIA NA MANUTENÇÃO DOS PROCESSOS EM DISTRIBUIDORAS DE

MATERIAL MÉDICO HOSPITALAR (MMH)

Paulo Augusto Bandeira Bernardino, Aline André da Silva Pereira, Amanda Gonçalves Pereira

produtos para diagnóstico de uso in vitro, buscando a garantia da qualidade, segurança e eficácia dos produtos no Brasil.

A RDC 16/2013 possui nove capítulos contendo as práticas que devem ser adotadas por esse segmento que envolvem: Disposições gerais; Requisitos Gerais do Sistema da Qualidade; Documentos e Registros da Qualidade; Controle de Projeto e Registro mestre de produto (rmp); Controles de Processo e Produção; Capítulo Manuseio, Armazenamento, Distribuição e Rastreabilidade; Ações Corretivas e Preventivas; Assistência Técnica; Técnicas Estatísticas. Dentro dessa resolução também nos deparamos com as Ações Corretivas e Preventivas que estão relacionadas com as auditorias.

Este estudo visa mostrar os impactos na manutenção dos processos de qualidade quando os programas de auditoria interna em distribuidoras de material médico hospitalar são realizados por profissionais desqualificados. Os auditores que não possuem conhecimento suficientes, tais como, saber quais registros devem ser cobrados na manutenção dos processos de qualidade, como realizar abordagem aos funcionários e analisar corretamente os procedimentos, terão dificuldades em identificar as não conformidades ou as oportunidades de melhorias. Quando isto acontece, os processos da empresa podem ser prejudicados.

A auditoria interna é de suma importância na manutenção dos processos exigidos pela RDC 16/2013. E quando ela é realizada de forma eficaz nas distribuidoras de $M M H$, percebe-se uma maior segurança, qualidade e garantia de eficácia referente aos produtos comercializados. Verificando se os requisitos da RDC 16/2013 estão sendo cumpridos através da auditoria interna, há uma contribuição para manutenção dos processos exigidos pela Agência Nacional de Vigilância Sanitária (ANVISA), além de maior qualidade nos processos, englobando todo ciclo de fornecimento aos hospitais e, consequentemente, trazendo maior eficácia aos pacientes.

Para Crepaldi (2000, p. 27), a auditoria foi definida como: "o levantamento, estudo e avaliação sistemática das transações, procedimentos, operações, rotinas e das demonstrações financeiras de uma entidade". Essas auditorias são realizadas para verificar a conformidade dos processos visando atender as expectativas exigidas por lei e o atendimento com excelência, ou seja, a auditoria é utilizada como ferramenta para monitoramento e manutenção dos processos organizacionais.

Para o autor Davenport (1994), "processo é uma ordem detalhada e específica das atividades de trabalho com tempo, espaço, um começo, um fim, inputs e outputs, todos bem identificados". Para os autores Hammer e Champy (1994), "processo são atividades realizadas em uma sequência racional com o objetivo de produzir bens ou serviços que agregam valor para os clientes específicos". O autor Gonçalves (1995) também define processo da seguinte maneira: "qualquer atividade ou um conjunto 


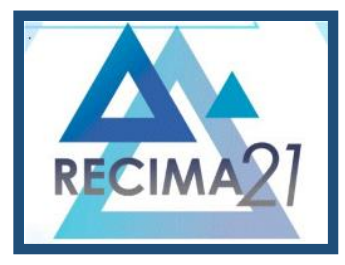

\section{RECIMA21 - REVISTA CIENTÍFICA MULTIDISCIPLINAR}

A IMPORTÂNCIA DA AUDITORIA NA MANUTENÇÃO DOS PROCESSOS EM DISTRIBUIDORAS DE MATERIAL MÉDICO HOSPITALAR (MMH) Paulo Augusto Bandeira Bernardino, Aline André da Silva Pereira, Amanda Gonçalves Pereira

delas que toma um input (entrada), adiciona valor e fornece um output (saída) a um cliente específico". Observa-se entre os autores, que os processos são atividades que agregam valor ao cliente.

\section{PROCESSOS EXIGIDOS PELA RDC 16/2013}

A Resolução da Diretoria Colegiada RDC 16/2013 (2013), publicada pela ANVISA, se refere a boas práticas que os distribuidores de $\mathrm{MMH}$ devem cumprir. Exige-se estabelecer e manter um sistema de qualidade para assegurar que os requisitos sejam atingidos e que os produtos fabricados sejam seguros, eficazes e adequados ao uso pretendido.

A gerência executiva deve estabelecer sua política e objetivos da qualidade, sendo esses mensuráveis e disponíveis a todos da organização por meio de um Manual da Qualidade. Requere estabelecer e manter estrutura organizacional adequada através de organograma e pessoal suficiente para assegurar o cumprimento do regulamento, definir responsabilidades, autoridades, funções, recursos adequados e designar pessoal treinado. Todas essas exigências devem ser registradas e armazenadas.

Devem ser assegurados os recursos do sistema da qualidade em conformidade com a RDC 16/2013. Uma das maneiras de controlar e monitorar o cumprimento dessa exigência é através das auditorias de qualidade, que também é uma obrigação descrita nas Ações Corretivas e Preventivas da norma.

No que diz respeito à avaliação e efetividade do sistema de qualidade, é requerido implantar uma revisão periódica para assegurar que são mantidas as normas. Nessa revisão um dos assuntos pertinentes é os resultados de auditorias e situações das ações corretivas e preventivas.

Segundo a norma ISO 9000:2005 ação corretiva é uma "ação para eliminar a causa de uma não conformidade identificada ou outra situação indesejável" (ISO 9000:2015, 2015, p. 15). Para Sterrit \& Nyblom (2007) ação corretiva é uma intervenção a um processo, a fim de evitar recorrência de um evento que resultou em perdas. Observa-se uma relação para ação corretiva, que serve para evitar uma não conformidade. De acordo com a norma, a "ação preventiva é executada para prevenir a ocorrência" (ISO 9000:2015, 2015, p. 33), ou seja, é utilizada visando não cometer as mesmas não conformidades.

Deve ser estabelecido e mantido procedimentos para o manuseio, armazenamento, distribuição e rastreabilidade dos produtos comercializados. Para Pozo (2010, p.11), "armazenagem é o processo que envolve administração dos espaços necessários para manter os materiais estocados". Diferente de Moura (2008), que define armazenagem como uma denominação da guarda dos produtos 


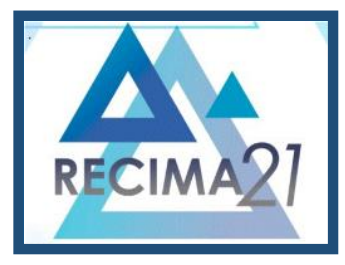

\section{RECIMA21 - REVISTA CIENTÍFICA MULTIDISCIPLINAR}

A IMPORTÂNCIA DA AUDITORIA NA MANUTENÇÃO DOS PROCESSOS EM DISTRIBUIDORAS DE MATERIAL MÉDICO HOSPITALAR (MMH) Paulo Augusto Bandeira Bernardino, Aline André da Silva Pereira, Amanda Gonçalves Pereira

temporariamente até a sua distribuição. A validade dos produtos estocados deve ser monitorada constantemente para que não haja utilização de materiais com prazo de validade expirado.

Nas Ações Corretivas e Preventivas da RDC 16/2013 é exigido análise dos processos, operações, reclamações, relatórios de auditoria com intuito de identificar causas existentes ou potenciais de não conformidade. As auditorias podem ser divididas em duas formas: (1) Auditoria Interna atuando nas verificações dos cumprimentos das normas e dos processos internos adotadas pela empresa realizada por um grupo de colaboradores treinados e capacitados para execução (CREPALDI, 2011); (2) Auditoria externa que é realizada por profissionais que não estão vinculados a empresa, atuam de forma independente e apresentam pareceres ou relatórios sobre o que está sendo auditada (BARRETO; GRAEFF, 2011).

Para análise das reclamações é importante citar que para a ANVISA (2013), reclamação é definida como "comunicação por escrito, oral ou eletrônica relativa à não aceitação da identidade, qualidade, durabilidade, confiabilidade, segurança, eficácia ou desempenho de um produto", onde é exigido estabelecer e manter procedimentos para receber, examinar, avaliar, investigar e arquivar as reclamações.

Nas técnicas estatísticas devem ser estabelecidos e mantidos processos para avaliar o desempenho do sistema de gestão da qualidade (SGQ), visando atender as especificações da resolução, através de técnicas estatísticas que devem ser sempre revisadas. Tais técnicas e as auditorias estão intimamente ligadas ao controle da qualidade que segundo Paladini (1990) é a comparação de resultados obtidos com os padrões ou objetivos pré-fixados. Já para Besterfield (1990) consiste no uso de técnicas e atividades para atingir, manter e melhorar a qualidade de um produto ou serviço.

Organizando os procedimentos de trabalho, tarefas e atividades é possível obter informações e observar pontos críticos, oportunidades de melhoria e mudanças. Portanto, o uso do controle estatístico da qualidade é essencial para que se tenha monitoramento. A norma estabelece que os resultados das auditorias sejam também analisados através dos indicadores.

\section{IMPORTÂNCIA DA AUDITORIA INTERNA NA MANUTENÇÃO DOS PROCESSOS EXIGIDOS PELA RDC 16/2013}

Conforme a RDC 16 ANVISA (2013) os distribuidores devem conduzir e documentar as auditorias da qualidade avaliando a conformidade do SGQ.

Para a ANVISA, auditoria de qualidade significa: um exame estabelecido, sistemático e independente de todo o sistema de qualidade de um fabricante, executado em intervalos regulares e com frequência suficiente para assegurar que 


\section{RECIMA21 - REVISTA CIENTÍFICA MULTIDISCIPLINAR}

A IMPORTÂNCIA DA AUDITORIA NA MANUTENÇÃO DOS PROCESSOS EM DISTRIBUIDORAS DE

MATERIAL MÉDICO HOSPITALAR (MMH)

Paulo Augusto Bandeira Bernardino, Aline André da Silva Pereira, Amanda Gonçalves Pereira

tanto as atividades do sistema de qualidade quanto seus resultados satisfaçam os procedimentos especificados em seu sistema de qualidade (ANVISA, 2016, p. 4).

De acordo com a norma ISO 9000:2005 (2005) as auditorias são usadas para determinar em que grau os requisitos do sistema de gestão da qualidade foram atendidos, avaliando a sua eficácia e identificando as oportunidades de melhoria. A auditoria deve ser planejada visando examinar todas as etapas estipuladas e a regularidade das eficiências dos processos e gestão da empresa. A auditoria também serve como parâmetro para identificar melhorias nos processos e controles internos da organização. $O$ auditor deve planejar como seu trabalho será conduzido e colher todas as evidências do seu trabalho de forma documentada, conforme exigido pela RDC.

A utilização das auditorias pode ser uma maneira de identificar oportunidades de melhoria visando um atendimento de excelência ao cliente, garantindo a credibilidade de seu atendimento no mercado. Os resultados gerados devem ser analisados criticamente para tratamento ou aperfeiçoamento do processo/produto, visando a melhoria contínua que, segundo a norma ISO 9000:2015 (2015), significa "atividade recorrente de para aumentar o desempenho".

O objetivo da auditoria é ter a eficiência das operações. Segundo Maximiano (2000, p 115), "eficiência realiza tarefas de maneira inteligente, com o mínimo de esforço e com o melhor aproveitamento possível dos recursos". Com a utilização das auditorias é visado a realização das atividades com excelência. Também pode-se verificar as não conformidades (não atendimento a um requisito), que devem ser tratadas com ações corretivas e, se possível, implementar ações preventivas que, conforme a norma ISO 9000:2015 (2015), são "executadas para prevenir a ocorrência, enquanto a ação corretiva é executada para prevenir a recorrência".

A auditoria é uma ferramenta de apoio à gestão que auxilia a instituição a controlar seus processos e atividades, atendendo as especificações estabelecidas. Todas as ações decididas para corrigir as falhas identificadas devem ser avaliadas quanto a sua efetividade. O monitoramento das ações se faz necessário até alcançar um grau satisfatório.

Em meio a competitividade e constantes mudanças, as empresas precisam aprimorar os seus desempenhos para garantir diferenciais competitivos visando a satisfação total do cliente. As auditorias são uma das formas que possibilitam identificar as falhas e oportunidades de melhorias presentes nas empresas, contribuindo assim para aprimoramento dos processos da qualidade e consequentemente agregando valor ao cliente.

\section{IMPACTOS NA MANUTENÇÃO DOS PROCESSOS DE QUALIDADE QUANDO REALIZADO POR PROFISSIONAIS DESQUALIFICADOS.}




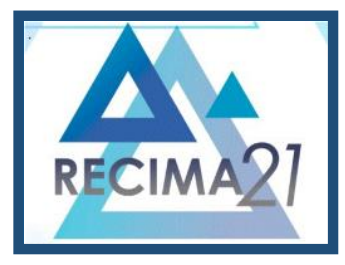

\title{
RECIMA21 - REVISTA CIENTÍFICA MULTIDISCIPLINAR
}

A IMPORTÂNCIA DA AUDITORIA NA MANUTENÇÃO DOS PROCESSOS EM DISTRIBUIDORAS DE
MATERIAL MÉDICO HOSPITALAR (MMH) Paulo Augusto Bandeira Bernardino, Aline André da Silva Pereira, Amanda Gonçalves Pereira

A empresa deve estabelecer um sistema de qualidade para certificar que todos os requisitos exigidos por ela ou por órgão externo sejam cumpridos. A auditoria interna auxilia a verificar o cumprimento de todos os procedimentos e processos. Mas, para isso, é necessário que os profissionais responsáveis pelas auditorias sejam treinados e capacitados.

Para Chiavenato:

\begin{abstract}
Modernamente, o treinamento é considerado um meio de desenvolver competências nas pessoas para que se tornem mais produtivas, criativas e inovadoras, a fim de contribuir melhor para os objetivos organizacionais e se tornarem cada vez mais valiosas. (CHIAVENATO, 2010, p. 367).

Treinamento: é o processo educacional de curto prazo e aplicado de maneira sistemática e organizada, por meio do qual as pessoas aprendem conhecimentos, atitudes e competências em função de objetivos previamente definidos. (CHIAVENATO, 2010, p. 367).
\end{abstract}

Os treinamentos são uma preparação essencial para a efetividade da verificação das não conformidades e oportunidades de melhoria, que devem ser realizados por profissionais previamente qualificados.

Quando as auditorias são realizadas por profissionais desqualificados, a efetividade dos processos não estará garantida. Não conformidades em processos e produtos podem não ser identificadas e consequentemente chegarão ao consumidor um atendimento fora do padrão de qualidade. Além do impacto para o cliente final, uma imagem ruim e consequências fiscais podem surgir.

Pensando nessas consequências, a própria norma RDC 16/2013 (2013) em seu requisito de Pessoal/Treinamento descreve que deve ser assegurado que todos os colaboradores sejam treinados para executar adequadamente as tarefas a ele designadas, conduzido de acordo com os procedimentos estabelecidos. Essas pessoas devem ser qualificadas para garantir que tenham uma compreensão de suas funções e dos requisitos da norma aplicados à função. Essa exigência se aplica aos auditores e demais funções da organização.

A RDC 16/2013 requisita que as auditorias devem ser conduzidas por pessoas comprovadamente treinadas de acordo com os procedimentos estabelecidos sem responsabilidade direta pelos itens que estão sendo objetos da auditoria. Basicamente o treinamento prepara o auditor para realizar as auditorias de forma adequada, visando buscar as conformidades dos processos. Sem o conhecimento referente às exigências da legislação não é possível realizar uma auditoria eficiente e eficaz.

É importante que as auditorias sejam realizadas por profissionais qualificados, pois estes garantem que as atividades desenvolvidas pela empresa estejam dentro dos requisitos estabelecidos. 


\section{RECIMA21 - REVISTA CIENTÍFICA MULTIDISCIPLINAR}

A IMPORTÂNCIA DA AUDITORIA NA MANUTENÇÃO DOS PROCESSOS EM DISTRIBUIDORAS DE MATERIAL MÉDICO HOSPITALAR (MMH) Paulo Augusto Bandeira Bernardino, Aline André da Silva Pereira, Amanda Gonçalves Pereira

\section{MÉTODOS PARA TRATAMENTO DE NÃO CONFORMIDADES}

Sabe-se que durante as auditorias internas ou externas podem ser encontradas não conformidades, que são prejudiciais à empresa, pois podem comprometer a qualidade do produto ou serviço. Como é definido por CAMPOS (1992), "um produto ou serviço de qualidade é aquele que atende perfeitamente, de forma confiável, de forma acessível, de forma segura e no tempo certo às necessidades do cliente".

Para a ISO 9000:2015, não conformidade é "o não atendimento de um requisito pré-estabelecido. Verifica-se uma relação com a não conformidade e a qualidade, pois estas devem atender a requisitos já estabelecidos, seja pela empresa ou pelo cliente.

Define-se como requisito: "condição básica e necessária para se obter alguma coisa ou para alcançar determinado propósito" (DICIO,2020). Esses requisitos podem vir de fatores externos, como normas de ISO, ou fatores internos, que são os processos e procedimentos determinados pela própria empresa.

A Gestão da qualidade contém várias ferramentas que podem ser utilizadas para tratamento de não conformidades e melhorias de processo. Dentre essas ferramentas, destacam-se as mais utilizadas: $5 \mathrm{~W} 2 \mathrm{H}$, Diagrama de Ishikawa, Matriz GUT, PDCA e Brainstorming. Para o tratamento de não conformidade, pode-se utilizar uma ou mais ferramentas ao mesmo tempo.

5W2H: Ferramenta da qualidade utilizada para conhecer uma não conformidade ou resolvê-la como plano de ação. São utilizadas 5 perguntas como base:

What (O que?): Qual a meta e objetivo? Qual estratégia e planejamento? Quais tarefas serão executadas?

Why (Por que?): Por que a etapa anterior necessita ser realizada? Traz algum benefício para a empresa?

Where (Onde?): Qual a área da empresa será responsável pela execução? Será interno ou externamente?

Who (Por quem?): Quem serão as pessoas responsáveis pela execução?

When (Quando?): Quando será o início e o término? Nessa etapa são determinados prazos.

How (Como?): Como o método será executado no desenvolver das atividades?

How much (Quanto?) Qual o gasto financeiro que a empresa deverá desembolsar para solução da não conformidade. 


\section{RECIMA21 - REVISTA CIENTÍFICA MULTIDISCIPLINAR}

A IMPORTÂNCIA DA AUDITORIA NA MANUTENÇÃO DOS PROCESSOS EM DISTRIBUIDORAS DE MATERIAL MÉDICO HOSPITALAR (MMH) Paulo Augusto Bandeira Bernardino, Aline André da Silva Pereira, Amanda Gonçalves Pereira

Diagrama de Ishikawa: Conhecido também pelo nome Diagrama de Espinho de Peixe, serve para investigar as não conformidades ou problemas que surgem na empresa, relacionando às possíveis causas ao seu efeito e levantando a causa raiz desses problemas. Ele é dividido da seguinte forma:

Variável que é definido como "aspectos, propriedades, características individuais ou fatores mensuráveis, através dos diferentes valores que assumem, discerníveis em um objeto de estudo, para testar a relação enunciada em uma proposição" Köche (2002, p.112). Por outro lado variável, é "uma classificação ou medida, uma quantidade que varia, um conceito operacional, que contém ou apresenta valores" Lakatos e Marconi (2003, p.175). Verifica-se uma posição intermediária que a variável deve ser mensurada, medida e apresentar valores.

Medida: Relacionado com as medidas, métricas e indicadores de qualidades. Falta de calibração de equipamentos, ausência de autorias e ou inspeções, métricas incorretas podem ser possíveis falhas de medida.

Material: Relacionado à matéria-prima ou insumo e o seu mau uso na área de trabalho e, até mesmo, à qualidade do material que é enviado pelo fornecedor.

Máquina: Relacionado à manutenção dos equipamentos, mau uso ou funcionamento inadequado.

Mão de obra: Relacionado às falhas das pessoas no processo das atividades.

Método: Relacionado aos meios utilizados pela empresa, como sistemas e procedimentos.

Meio ambiente: Relacionado ao local de trabalho dos colaboradores, funcional para o exercício das atividades.

Matriz GUT: Ferramenta utilizada na priorização das não conformidades, ou seja, qual ou quais não conformidades terão prioridades no tratamento. GUT significa Gravidade, Urgência e Tendência, esses recebem pontuações de 1 a 5 conforme análise e nível de impacto para a organização.

Gravidade: Leva em consideração impacto que a empresa pode sofrer caso essa não conformidade não seja tratada.

Urgência: Leva em consideração quão urgente é a tratativa da não conformidade.

Tendência: Leva em consideração a evolução da não conformidade caso ela não seja tratada. 


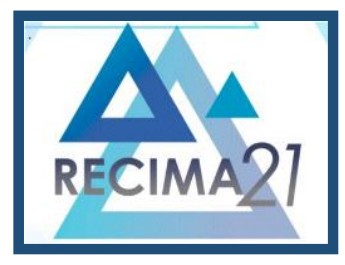

\section{RECIMA21 - REVISTA CIENTÍFICA MULTIDISCIPLINAR}

A IMPORTÂNCIA DA AUDITORIA NA MANUTENÇÃO DOS PROCESSOS EM DISTRIBUIDORAS DE MATERIAL MÉDICO HOSPITALAR (MMH) Paulo Augusto Bandeira Bernardino, Aline André da Silva Pereira, Amanda Gonçalves Pereira

PDCA: Método para melhoria de produtos, processos ou serviços. O PDCA é descrito por Moura $(1197$, p.90) como "uma ferramenta que orienta a sequência de atividades para gerenciar uma tarefa, processo, empresa etc.". Nesse método é identificado um problema e utilizado uma meta para resolvê-lo. Esse método pode ser realizado uma vez ou diversas vezes sobre a mesma não conformidade, depende do resultado que será encontrado no final do ciclo. Esse método é dividido em quatro etapas: Plan (planejar), Do (executar), Check (Chegar) e Act (agir).

Plan (Planejar): Nessa etapa o problema ou não conformidade será conhecido, com a suas possíveis causas identificadas, com os dados disponíveis sendo analisados, estabelecendo metas para a resolução do problema e definindo planos de ação para alcançar o objetivo.

Do (Executar): Nessa etapa será executado o que foi planejado na fase do planejamento.

Check (Checar): Nessa etapa será feita a verificação e análise de todos os dados coletados da fase anterior.

Act (Agir): Nessa etapa poderão ser realizados possíveis alterações, como recomeçar o ciclo caso as etapas anteriores não tenham obtido sucesso ou caso alguma conformidade não tenha sido identificada conforme o planejado. O ciclo pode ser refeito para cada nova meta estabelecida.

Brainstorming: É uma técnica em grupo para identificar causas e soluções para não conformidades ou levantar ideias para melhorias da empresa. Nessa reunião são utilizadas pessoas de vários setores que possam contribuir efetivamente para o assunto proposto.

\section{INFLUÊNCIA DOS PROGRAMAS DE AUDITORIA INTERNA AOS HOSPITAIS E PACIENTES}

Sabe-se que a auditoria interna é fundamental para as empresas, independente do ramo de atuação. Entretanto, para as distribuidoras de produtos médicos hospitalares, verifica-se um cuidado especial, visto finalidade dos produtos à saúde humana e caso esses produtos não estejam de acordo com os critérios estabelecidos, hospitais e pacientes poderão ser gravemente prejudicados.

A empresa deve estabelecer um sistema de qualidade para certificar que os requisitos exigidos sejam cumpridos. A auditoria interna auxilia a verificar o cumprimento de todos os procedimentos conforme estabelecido pela RDC 16/2013. Tanto no fabricante quanto nas distribuidoras, os requisitos devem ser cumpridos para manter a qualidade dos produtos e processos, sendo estes eficientes e eficazes para os hospitais e pacientes.

As distribuidoras de $\mathrm{MMH}$ devem certificar-se que seus fornecedores sejam idôneos e em conformidade com a RDC. Para este tipo de certificação é necessário que seja realizada auditoria in 


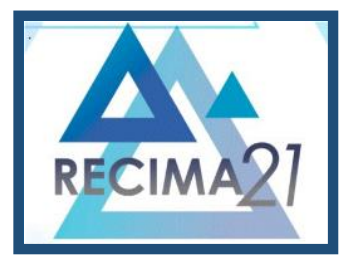

\section{RECIMA21 - REVISTA CIENTÍFICA MULTIDISCIPLINAR}

A IMPORTÂNCIA DA AUDITORIA NA MANUTENÇÃO DOS PROCESSOS EM DISTRIBUIDORAS DE MATERIAL MÉDICO HOSPITALAR (MMH) Paulo Augusto Bandeira Bernardino, Aline André da Silva Pereira, Amanda Gonçalves Pereira loco de qualificação nas dependências da empresa. Essa auditoria deve seguir critérios estabelecidos pela ANVISA, e o fornecedor deve possuir o Certificado de Autorização de Funcionamento (AFE) e o Certificado de Boas Práticas de Fabricação (CBPF), comprovando que a empresa está autorizada a exercer suas atividades. Deve ser emitido um relatório sobre os resultados dessa auditoria e manter documentado.

Os fornecedores devem certificar a procedência dos materiais que são utilizados em sua cadeia produtiva, bem como os registros de todos os pedidos de compra, insumos, certificação de qualidade, aprovação dos pedidos e o comprometimento dos fabricantes caso algum insumo sofra alteração. Todos os documentos devem ser aprovados, revisados e armazenados. Além desses dados, os registros de inspeções e testes também deverão ser mantidos para certificar a qualidade do produto, a aceitação e a aprovação.

Fornecedores "são unidades que embora constituídas, tal como as outras, com meios humanos, materiais e monetários - atuam na lógica das leis do mercado ou do plano e são condicionadas por variáveis ambientais que interagem com seu desenvolvimento" (LISBOA et al., 2007, p.14). Os fornecedores e os distribuidores de produtos $\mathrm{MMH}$ têm a finalidade de abastecer os lugares que realizam atendimentos com os materiais necessários para cada tipo de procedimento. É fundamental que eles atendam de forma segura e forneçam produtos com boa procedência, tecnologia e excelência na qualidade, visando o objetivo final que é a recuperação do paciente.

Observa-se que além dos cuidados com os fabricantes, a qualidade dos produtos, o controle e monitoramento dos processos internos das distribuidoras precisam ser seguros e em conformidade com as especificações da RDC. Desde a instalação das dependências da empresa, sua limpeza e organização, detalhamentos das operações, higiene pessoal dos empregados e equipamentos para produção, tudo deve ser feito para garantir as conformidades de especificações. Além disso, os fabricantes devem realizar processo de gerenciamento de riscos, que está relacionado com ciclo de vida do produto, desde o início até o fim da vida útil. Este gerenciamento identifica possíveis riscos que devem ser controlados, avaliados e seus controles efetivamente estabelecidos.

Os controles referentes aos produtos não são limitados somente aos insumos para o produto acabado e a sua produção, mas estende-se às embalagens, rótulos, instruções de uso, calibração e manutenção de todos os equipamentos referente às inspeções. Qualquer alteração deve ser requisitada, documentada e aprovada. Outro controle não menos importante está relacionado com os sistemas de informática, softwares, equipamentos, métodos ou qualquer alteração que possa impactar na qualidade do produto. 


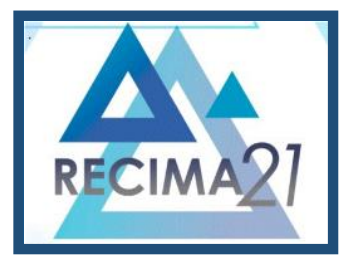

\section{RECIMA21 - REVISTA CIENTÍFICA MULTIDISCIPLINAR}

A IMPORTÂNCIA DA AUDITORIA NA MANUTENÇÃO DOS PROCESSOS EM DISTRIBUIDORAS DE MATERIAL MÉDICO HOSPITALAR (MMH) Paulo Augusto Bandeira Bernardino, Aline André da Silva Pereira, Amanda Gonçalves Pereira

Outro fator importante é o manuseio e armazenamento dos produtos, com controle para que defeitos como, por exemplo, deterioração e danos não ocorram durante essa fase. Na distribuição, registros como nome, endereço, identificação, quantidade, data de expedição e qualquer identificação que possa ajudar na rastreabilidade deverão constar nos controles dos fabricantes, fornecedores e distribuidores.

Caso em algum processo seja encontrada não conformidade, os controles deverão garantir que esses produtos não sejam utilizados. Eles devem ser identificados, avaliados, documentados e segregados como não conforme, necessitando de notificação e investigação das pessoas envolvidas em tratar esses problemas de acordo com o processo da empresa. Todas as etapas devem ser documentadas conforme os requisitos estipulados. Todas as causas que possam estar relacionadas à não conformidade deverão ser analisadas, investigadas e registradas, bem como as execuções de ações para prevenir e corrigir as ocorrências.

$\mathrm{Na}$ realização da escolha dos materiais médicos hospitalares, algumas análises devem ser realizadas e levadas em consideração e não somente as informações sobre o produto que está sendo adquirido, mas também verificar quem sãos os fornecedores desses produtos e se eles possuem qualificação no setor da saúde para a distribuição.

No processo gerencial da comercialização de produtos médicos hospitalares observa-se a corresponsabilidade entre fabricantes, distribuidores e hospitais ao trabalharem com esse tipo de material, uma vez que a qualificação dessas instituições deve ser realizada a fim de assegurar que o produto esteja em conformidade com suas especificações e dentro dos níveis de qualidade exigidos pelos órgãos responsáveis, como, por exemplo, a brasileira ANVISA. Ela garantirá o cumprimento dos requisitos exigidos (auditorias internas e externas) dentro dos processos de comercialização dos produtos médicos hospitalares.

Seja na fabricação ou na distribuição dos produtos, a empresa deverá estabelecer normas e procedimentos para que os produtos não conformes não sejam utilizados em hospitais ou pacientes. Considerando que esses procedimentos estão sendo cumpridos e analisados, obtêm- se um melhor resultado.

\section{CONSIDERAÇÕES FINAIS}

O problema de pesquisa desse projeto indagou a importância da auditoria interna para manutenção dos processos exigidos pela RDC 16/2013 (ANVISA). Extrapolando o pressuposto inicial, verificou-se 


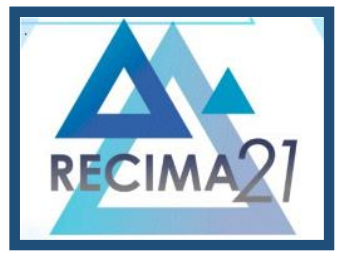

\section{RECIMA21 - REVISTA CIENTÍFICA MULTIDISCIPLINAR}

A IMPORTÂNCIA DA AUDITORIA NA MANUTENÇÃO DOS PROCESSOS EM DISTRIBUIDORAS DE MATERIAL MÉDICO HOSPITALAR (MMH) Paulo Augusto Bandeira Bernardino, Aline André da Silva Pereira, Amanda Gonçalves Pereira que os requisitos exigidos na resolução são de suma importância para o bom funcionamento das distribuidoras de $\mathrm{MMH}$ e o seu monitoramento pode ser feito através das auditorias.

A partir do objetivo da auditoria exposto, conclui-se que os programas de auditoria interna em distribuidoras de $\mathrm{MMH}$ impactam nos hospitais e clientes que são atendidos com os processos e produtos em conformidade, evitando riscos e contribuindo para o bem-estar dos pacientes, garantindo assim, a credibilidade perante o mercado.

Conclui-se, ainda, que a auditoria interna garante ao distribuidor uma verificação de se os procedimentos estão sendo cumpridos, contribuindo também para visualização de oportunidades de melhoria e aperfeiçoamento. Além disso, as auditorias devem ser realizadas por profissionais qualificados e preparados, visando a eficiência nessa inspeção. $\mathrm{O}$ conhecimento referente à norma $\mathrm{e}$ as técnicas para tratamento de não conformidades são essenciais para garantir uma auditoria de qualidade.

\section{REFERÊNCIAS}

AGÊNCIA NACIONAL DE VIGILANCIA SANITÁRIA. Resolução da Diretoria Colegiada $\mathbf{n}^{\circ} \mathbf{1 6}$, de 28 de março de 2013. Aprova o Regulamento Técnico de Boas Práticas de Fabricação de Produtos Médicos e Produtos para Diagnostico de Uso IN Vitro e dá outras providencias. Disponível em: http://bvsms.saude.gov.br/bvs/saudelegis/anvisa/2013/rdc0016 2803 2013.pdf. Acesso em: 16 mar. 2020.

ASSOCIAÇÃO BRASILEIRA DE NORMAS TECNICAS (2005). Norma Brasileira ISO 9000:2005. Disponível em: https://qualidadeuniso.files.wordpress.com/2012/09/nbr-iso-9000-2005.pdf. Acesso em: 20 maio 2020.

ALONÇO, Guilherme. $O$ que é não conformidade? Como tratar uma NC?. Campinas: Templum. Disponível em: https://certificacaoiso.com.br/o-que-e-nao-conformidade/. Acesso em: 13 maio 2020.

ANDRADE, Fernanda Beazi; SICHESKI, Sirineu José. Auditoria interna hospitalar: uma atividade de apoio à tomada de decisão. Disponível em: https://www.revistaespacios.com/a17v38n24/17382413.html. Acesso em: 08 maio 2020.

BARRETO, D. E.; GRAEFF, F. Auditoria: teoria e exercícios comentados. 2. ed. São Paulo: Método, 2011.

BESTERFIELD, D. H. Gerenciamento da qualidade total. Nova Jersey: Prentice-Hall, 1995.

CAMPOS, V. FALCONI. Gerenciamento da Rotina do Trabalho do Dia a Dia. 6. ed. Belo Horizonte: Editora de Desenvolvimento Gerencial, 1994.

CAMPOS, V. FALCONI, T. Q. C. Controle da Qualidade Total (no estilo japonês). Belo Horizonte: Fundação Cristiano Otoni/Escola de Engenharia da Universidade Federal de Minas Gerais, 1992. 


\section{RECIMA21 - REVISTA CIENTÍFICA MULTIDISCIPLINAR}

A IMPORTÂNCIA DA AUDITORIA NA MANUTENÇÃO DOS PROCESSOS EM DISTRIBUIDORAS DE MATERIAL MÉDICO HOSPITALAR (MMH) Paulo Augusto Bandeira Bernardino, Aline André da Silva Pereira, Amanda Gonçalves Pereira

CHIAVENATO, Idalberto. Gestão de Pessoas: o novo papel dos recursos humanos nas organizações. 3. ed. Rio de Janeiro: Elsevier, 2010.

CREPALDI, Silvio Aparecido. Auditoria Contábil: teoria e prática. São Paulo: Atlas, 2000.

CREPALDI, Silvio Aparecido. Auditoria Contábil: teoria e prática. 7. ed. São Paulo: Atlas, 2011.

DAVENPORT, Thomas H. Reengenharia de processos. Rio de Janeiro: Campus, 1994.

ABNT NBR ISO 9000 Sistemas de gestão da qualidade. Disponível em: https://www.academia.edu/32163836/ABNT NBR ISO 9000 Sistemas de gest\%C3\%A30 da quali dade Fundamentos e vocabul\%C3\%A1rio Quality management systems Fundamentals and voC abulary. Acesso em: 08 jun. 2020.

GALO, Gisele Cristiane; BARBOSA, Roseli Aparecida de Oliveira. Auditoria Interna e Externa. Disponível em: http://www.univale.com.br/portalnovo/images/root/anaisadmix/5.pdf. Acesso em: 08 maio 2020.

GESTÃO OPME. 6 pontos importantes que você deve conhecer sobre a RDC 16/2013. Disponível em: https://gestaoopme.com.br/6-pontos-importantes-que-voce-deve-conhecer-sobre-ardc-16-2013. Acesso em: 14 mar. 2020.

GONÇALVES, J. E. L.; DREYFUS, Cassio. Reengenharia das empresas: passando a limpo. São Paulo: Atlas, 1995.

HAMMER, Michael; CHAMPY, James. Reengenharia da corporação. New York: HarperBusiness, 1994.

INSTITUTO ANTONIO HOUAISS. Dicionário eletrônico da língua portuguesa 1.0. Rio de Janeiro, 2001. 1 CD-ROM.

JUNIOR, Carlos. Ciclo PDCA: uma ferramenta imprescindível ao gerente de projetos. Disponível em: https://www.projectbuilder.com.br/blog/ciclo-pdca-uma-ferramenta-imprescindivel-ao-gerente-deprojetos/. Acesso em: 13 maio 2020.

JURAN, J. M. A qualidade desde o projeto: novos passos para o planejamento da qualidade em produtos e serviços. 3. ed. São Paulo: Pioneira, 1997. 551 p.

KÖCHE, J. C. Fundamentos de metodologia científica: teoria da ciência e iniciação à pesquisa. 20. ed. Petrópolis: Vozes, 2002. 182 p.

LAKATOS, E. M.; MARCONI, M. A. Metodologia científica. 5. ed. São Paulo: Atlas, 2008. 175 p.

LAS CASAS, Alexandre L. Qualidade total em serviços: conceitos, exercícios, casos práticos. 3. ed. São Paulo: Atlas, 1999. 206 p.

LIMA, Leandra de Jesus; LIMA; Robernei Aparecido. A importância da auditoria interna das organizações. Disponível em: http://www.portaldecontabilidade.com.br/tematicas/auditoria-internaorganizacoes.htm. Acesso em: 08 maio 2020.

LISBOA, J.; COELHO, A.; COELHO, F.; ALMEIDA, F. Introdução à gestão de Organizações. Barcelos: Vida Económica, 2007. 


\section{RECIMA21 - REVISTA CIENTÍFICA MULTIDISCIPLINAR}

A IMPORTÂNCIA DA AUDITORIA NA MANUTENÇÃO DOS PROCESSOS EM DISTRIBUIDORAS DE

MATERIAL MÉDICO HOSPITALAR (MMH) Paulo Augusto Bandeira Bernardino, Aline André da Silva Pereira, Amanda Gonçalves Pereira

LOCHINI, Thiago. Gestão Estratégica da qualidade. Disponível em: https://administradores.com.br/artigos/gestao-estrategica-da-qualidade. Acesso em 13 maio 2020.

MARQUES, Juracy C.; MIRSHAWKA, Victor. Luta pela qualidade a vez do Brasil ênfase no setor público. São Paulo: MAKRON Books, 1993. 287 p.

MARSHALL JUNIOR, Isnard; CIERCO, Agliberto Alves; ROCHA, Alexandre Varanda. Gestão da qualidade. 9. ed. São Paulo: Atlas, 2008. 201 p.

MAXIMIANO, Antonio Cesar Amaru. Introdução à Administração. 5. ed. São Paulo: Atlas. 2000. $115 \mathrm{p}$.

MOURA, L. R. Qualidade simplesmente total: uma abordagem simples e prática da gestão da qualidade. Rio de Janeiro: Qualitymark, 1997.

MOURA, Reinaldo A. Armazenagem: do Recebimento à Expedição em Almoxarifados ou Centros de Distribuição. São Paulo: IMAN, 2008.

PALADINI, Edson Pacheco. Qualidade total na prática: implantação e avaliação de sistemas de qualidade total. 2. ed. São Paulo: Atlas, 1997. 217 p.

PALADINI, E. P. Controle de Qualidade: uma abordagem abrangente. São Paulo: Editora Atlas, 1990.

PEINADO, Jurandir; GRAEML, Alexandre Reis. Administração da produção: operações industriais e de serviços. Curitiba: UnicenP, 2007.

POZO, Hamilton. Administração de Recursos Materiais e Patrimoniais: uma abordagem logística. São Paulo: Atlas, 2010. p. 11.

DICIO. Dicionário Online de Português. Disponível em: https://www.dicio.com.br/requisito/. Acesso em 04 jun. 2020.

RODRIGUES, Gleison. A Aplicação da Auditoria Interna nos Processos de Exportação no Ramo Madeireiro. Disponível em: https://www.aedb.br/seget/arquivos/artigos13/1411898.pdf. Acesso em 16 mar. 2020.

STERRIT, J. W.; NYBLOM, S. E. Planos de ação corretiva: Desenvolvendo e gerenciando o processo. Segurança Profissional, v. 52, n.10, p. 34-41, 2007. 